\title{
Fuzzy Multilevel Graph Embedding for Recognition, Indexing and Retrieval of Graphic Document Images
}

\author{
Muhammad Muzzamil Luqman \\ L3i Laboratory, University of La Rochelle, Avenue Michel Crépeau, 17042 La Rochelle, France \\ Advisors: Jean-Yves Ramel, Josep Lladós and Thierry Brouard \\ Date and location of PhD thesis defense: $2^{\text {nd }}$ of March 2012 at University of Tours in France \\ Received $15^{\text {th }}$ January 2014; accepted $25^{\text {th }}$ May 2014
}

\begin{abstract}
A pattern recognition task for computers can be looked upon as consisting of two main steps - (i) the representation of the signal data by a data structure and (ii) the computation of desired operation (pattern recognition). The two important sub domains of pattern recognition - the structural pattern recognition and the statistical pattern recognition - each have its strength only in one of the two aforementioned steps, respectively.

The structural pattern recognition offers the most powerful relational data structure of graph. For the last three decades, graphs have been used for pattern recognition and image analysis, for extracting and representing complex relations in underlying data. However, there is still a lack of efficient computational tools and learning models which can process this data structure.
\end{abstract}

On the other side, the statistical pattern recognition offers highly efficient computational models of machine learning, classification and clustering, by employing the well established theory of statistics. But these computational models can work only on simple numeric vectors and can not process complex high dimensional relational data structures.

Over decades of parallel research in both of these sub domains of pattern recognition - structural and statistical pattern recognition - powerful representations and efficient computational models have been built. But little progress has been made towards the long desired objective (of research in pattern recognition), to join the advantages of the structural and statistical pattern recognition approaches for building more powerful and efficient algorithms.

This thesis is a step forward to achieve this objective of joining the advantages of structural and statistical pattern recognition approaches. We propose an algorithm which permits the pattern recognition applications to employ the powerful relational data structure of attributed graphs along with the computational strengths of

Correspondence to: <muhammad_muzzamil.luqman@univ-lr.fr >

Recommended for acceptance by $<$ Alicia Fornés and Volkmar Frinken $>$

ELCVIA ISSN:1577-5097

Published by Computer Vision Center / Universitat Autònoma de Barcelona, Barcelona, Spain 
state of the art statistical models of machine learning, classification and clustering.

This thesis addresses the problem of lack of efficient computational tools for graph based structural pattern recognition approaches and proposes to exploit computational strength of statistical pattern recognition. The contribution of this thesis is two-fold.

The first contribution of this thesis is a new method of explicit graph embedding. The proposed graph embedding method exploits multilevel analysis of graph for extracting graph level information, structural level information and elementary level information from graphs. It embeds this information into a numeric feature vector. The method employs fuzzy overlapping trapezoidal intervals for addressing the noise sensitivity of graph representations and for minimizing the information loss while mapping from continuous graph space to discrete vector space. The method has unsupervised learning abilities and is capable of automatically adapting its parameters to underlying graph dataset.

The second contribution of this thesis is a framework for automatic indexing of graph repositories for graph retrieval and subgraph spotting. The proposed framework exploits explicit graph embedding together with classification and clustering tools. It achieves the automatic indexing of a graph repository during its off-line learning phase, where its extracts the cliques of order 2 from graphs and embeds them into feature vectors by employing the aforementioned explicit graph embedding technique. It clusters the feature vectors into classes, learns a classifier and builds an index for the graph repository. During on-line spotting phase, it extracts the cliques of order 2 from query graph, embeds them into feature vectors and uses the index of the graph repository to retrieve the graphs from repository. The framework does not require a labeled learning set and can be easily deployed to a range of application domains, offering ease of query by example (QBE) and granularity of focused retrieval.

Experimentation on latest public graph datasets from International Association of Pattern Recognition's Technical Committee on graph-based representations (TC-15) evaluates the power and applicability of our graph embedding framework for the problems of graph classification and graph clustering. A second set of experimentation evaluates the framework for automatic indexing of graph repositories for graph retrieval and subgraph spotting.

Applications to the real problems of recognition, indexing and retrieval of graphic document images are also presented.

Key Words: Pattern recognition, graph clustering, graph classification, graph embedding, subgraph spotting, fuzzy logic, graphics recognition.

\section{References}

[1] M.M. Luqman, J.-Y. Ramel, J. Llados, and T. Brouard, Fuzzy multilevel graph embedding, Pattern Recognition, 46(2):551-565, 2013.

[2] M.M. Luqman, Fuzzy Multilevel Graph Embedding for Recognition, Indexing and Retrieval of Graphic Document Images, LAMBERT Academic Publishing, ISBN 978-3- 659-49407-9, 2013.

[3] M.M. Luqman, Fuzzy Multilevel Graph Embedding for Recognition, Indexing and Retrieval of Graphic Document Images, $\mathrm{PhD}$ thesis, University of Tours (France) and Autonoma University of Barcelona (Spain), 2012. 